Pacific Journal of 


\title{
ON THE CONVERGENCE OF RESOLVENTS OF OPERATORS
}

\author{
Minoru Hasegawa
}

Let a family of linear operators $\left\{A_{n}\right\}(n=1,2, \cdots)$ in a Banach space $X$ have the resolvents $\left\{R\left(\lambda ; A_{n}\right)\right\}$ which is equicontinuous in $n$. Suppose that $\left\{A_{n}\right\}$ is a Cauchy sequence on a dense set. Then the question of convergence arises; when will $\left\{R\left(\lambda ; A_{n}\right) x\right\}$ be a Cauchy sequence for all $x \in X$ ?

This problem is treated in some special cases and an application to the following theorem is presented.

Let $A$ be the generator of a positive contraction semigroup $\sum$ and let $B$ be a linear operator with domain $\mathscr{D}(B)$ $\supset \mathscr{D}(A)$ in a weakly complete Banach lattice $X$.

Then $A+B$ or its closed extension generates a positive contraction semi-group $\Sigma^{\prime}$ which dominates $\sum$ if and only if $A+B$ is dissipative and $B$ is positive.

In this section we consider the above convergence problem in a Banach space $X$ (cf. [9], [1], [11]).

Let a family of linear operators $\left\{A_{n}\right\}(n=1,2, \cdots)$ satisfy the following conditions:

(1) for some fixed number $\lambda$, the resolvent $R\left(\lambda ; A_{n}\right)=\left(\lambda-A_{n}\right)^{-1}$ of $A_{n}$ exists which acts on $X$ to the domain $\mathscr{D}\left(A_{n}\right)$ of $A_{n}$ and satisfies the norm condition $\left\|R\left(\lambda ; A_{n}\right)\right\| \leqq K_{\lambda}$, where $K_{\lambda}$ is a positive number independent of $n$,

(2) there is a dense subspace $\mathscr{C}$ on which $A=\lim A_{n}$ exists.

Proposition 1. The limit operator $R_{0}(\lambda ; A)=\lim R\left(\lambda ; A_{n}\right)$ exists on $\overline{\mathscr{N}}$ and satisfies the norm condition $\left\|R_{0}(\lambda ; A)\right\|_{\overline{\mathscr{N}}} \leqq K_{\lambda}$ where $\mathscr{N}=$ $(\lambda-A) \mathscr{M}$ and $\overline{\mathscr{N}}$ is its closure.

Proof. For any $x \in \mathscr{L}$ we have

$$
\left\|\left(\lambda-A_{n}\right) x\right\| \geqq K_{\lambda}^{-1}\|x\|
$$

and thus obtain

$$
\|(\lambda-A) x\| \geqq K_{\lambda}^{-1}\|x\|-\left\|A_{n} x-A x\right\| .
$$

Letting $n \rightarrow \infty$, we have

$$
\|(\lambda-A) x\| \geqq K_{\lambda}^{-1}\|x\| \text {. }
$$

It also follows that we can extend $(\lambda-A)^{-1}$ to the bounded linear operator $R_{0}(\lambda ; A)$ on $\overline{\mathscr{N}}$ which satisfies 


$$
\left\|R_{0}(\lambda ; A)\right\|_{\bar{N}}=\sup \left\{\left\|R_{0}(\lambda ; A) x\right\| ;\|x\|=1, x \in \overline{\mathscr{N}}\right\} \leqq K_{\lambda} .
$$

Further, it is easy to see that, for any $x \in \mathscr{C}$,

$$
\left\|R\left(\lambda ; A_{n}\right)(\lambda-A) x-x\right\| \leqq K_{\lambda}\left\|A_{n} x-A x\right\|
$$

which implies that $R_{0}(\lambda ; A)=\lim R\left(\lambda ; A_{n}\right)$ on $\overline{\mathscr{N}}$.

REMARK 1. This proof shows that if $(\lambda-A) \mathscr{C}$ is dense in $X$ then the convergence problem is solved.

We next remark some modification of the basic lemma in [1].

Proposition 2. The following conditions are equivalent.

(1) $\lim _{n, n^{\prime} \rightarrow \infty}\left\|R\left(\lambda ; A_{n}\right) x-R\left(\lambda ; A_{n^{\prime}}\right) x\right\|=0 \quad(x \in X)$,

(2) $\lim _{n, n^{\prime} \rightarrow \infty}\left\|R\left(\lambda ; A_{n}\right) R\left(\lambda ; A_{n^{\prime}}\right) x-R\left(\lambda ; A_{n^{\prime}}\right) R\left(\lambda ; A_{n}\right) x\right\|=0$

$$
(x \in(\lambda-A) \mathscr{M}) \text {. }
$$

Proof. For any $x \in \mathscr{l}, n$ and $n^{\prime}$, we have

$$
\begin{aligned}
& R\left(\lambda ; A_{n}\right) x-R\left(\lambda ; A_{n^{\prime}}\right) x \\
&= R\left(\lambda ; A_{n}\right) R\left(\lambda ; A_{n^{\prime}}\right)\left(\lambda-A_{n^{\prime}}\right) x-R\left(\lambda ; A_{n^{\prime}}\right) R\left(\lambda ; A_{n}\right)\left(\lambda-A_{n}\right) x \\
&= R\left(\lambda ; A_{n}\right) R\left(\lambda ; A_{n^{\prime}}\right)(\lambda-A) x-R\left(\lambda ; A_{n^{\prime}}\right) R\left(\lambda ; A_{n}\right)(\lambda-A) x \\
&+R\left(\lambda ; A_{n}\right) R\left(\lambda ; A_{n^{\prime}}\right)\left(A-A_{n^{\prime}}\right) x \\
&+R\left(\lambda ; A_{n^{\prime}}\right) R\left(\lambda ; A_{n}\right)\left(A_{n}-A\right) x .
\end{aligned}
$$

From this relation and $\overline{\mathscr{C}}=X$, the assertion is readily verified.

Proposition 3. If, for some positive integer $m$,

$$
\lim _{n \rightarrow \infty}\left\|\left\{\left(A_{n}-A\right) R\left(\lambda ; A_{n}\right)\right\}^{m} x\right\|=0 \quad\left(x \in \mathscr{C}_{1}\right)
$$

is satisfied, where $\mathscr{C}_{1}$ is dense in $X$, then $(\lambda-A) \mathscr{C}$ is dense in $X$.

Proof. By virtue of the Hahn-Banach extension theorem, if there exists $x_{0} \in \mathscr{M}_{1}-\overline{\mathscr{N}}$, then so does a bounded linear functional $F_{0}$ acting on $X$ which satisfies the following conditions:

$$
F_{0}\left(x_{0}\right) \neq 0, \quad F_{0}(x)=0 \quad(x \in \overline{\mathscr{N}}=\overline{(\lambda-A) \mathscr{M})} .
$$

For this $x_{0}$ and any $n$, we have

$$
\begin{aligned}
x_{0}= & \left(\lambda-A_{n}\right) R\left(\lambda ; A_{n}\right) x_{0} \\
= & (\lambda-A) R\left(\lambda ; A_{n}\right) x_{0}-\left(A_{n}-A\right) R\left(\lambda ; A_{n}\right) x_{0} \\
= & \cdots \\
= & (\lambda-A) R\left(\lambda ; A_{n}\right) x_{0}-(\lambda-A) R\left(\lambda ; A_{n}\right)\left(A_{n}-A\right) R\left(\lambda ; A_{n}\right) x_{0} \\
& +-\cdots \\
& +(-1)^{m}\left\{\left(A_{n}-A\right) R\left(\lambda ; A_{n}\right)\right\}^{m} x_{0} .
\end{aligned}
$$


This relation implies that

$$
F_{0}\left(x_{0}\right)=(-1)^{m} F_{0}\left(\left\{\left(A_{n}-A\right) R\left(\lambda ; A_{n}\right)\right\}^{m} x_{0}\right)
$$

and for any $n$

$$
0<\left|F_{0}\left(x_{0}\right)\right| \leqq\left\|F_{0}\right\|\left\|\left\{\left(A_{n}-A\right) R\left(\lambda ; A_{n}\right)\right\}^{m} x_{0}\right\|
$$

which is a contradiction. Consequently we have $\mathscr{L}_{1} \subset \bar{N}$ and the assertion is proved.

We now concern with a theorem on the perturbation of operators which will be required in the sequel.

Proposition 4. Suppose that linear operators $A$ and $B$ satisfy the following conditions:

(1) for some number $\lambda$, the equation

$$
(\lambda-A) y=x \quad(x \in X)
$$

has a unique solution $y=R(\lambda ; A) x$,

(2) there is a dense subspace $\mathscr{C}$ such that $B R(\lambda ; A) \mathscr{C} \subset \mathscr{C}$ and

$$
\lim _{k \rightarrow \infty}\left\|\{B R(\lambda ; A)\}^{k} x\right\|=0 \quad(x \in \mathscr{C l}) .
$$

Then $(\lambda-A-B) R(\lambda ; A) \mathscr{l l}$ is dense in $X$.

The proof of this proposition is similar as that of Proposition 3 and is omitted.

REMARK 2. Suppose that for some positive integer $k$

$$
\left\|\{B R(\lambda ; A)\}^{k}\right\|_{\mathscr{N}}<1
$$

is satisfied, then the condition $(*)$ in Proposition 4 is satisfied.

REMARK 3. Suppose that $R(\lambda ; A)$ satisfies the norm condition $\|R(\lambda ; A)\| \leqq K_{\lambda}$ in Proposition 4 and that there exist positive constants $a$ and $b$ such that for any $x \in \mathscr{C}_{1}=R(\lambda ; A) \mathscr{C l}$

$$
\|B x\| \leqq a\|A x\|+b\|x\|
$$

and

$$
a|\lambda| K_{\lambda}+a+b K_{\lambda}<1 .
$$

Then the condition (**) in Remark 2 is satisfied.

Proof. For any $x \in \mathscr{A l}$, we have 


$$
\begin{aligned}
\|B R(\lambda ; A) x\| & \leqq a\|A R(\lambda ; A) x\|+b\|R(\lambda ; A) x\| \\
& \leqq a\|\lambda R(\lambda ; A) x-x\|+b K_{\lambda}\|x\|
\end{aligned}
$$

and

$$
\|B R(\lambda ; A) x\| \leqq\left(a|\lambda| K_{\lambda}+a+b K_{\lambda}\right)\|x\|<\|x\| .
$$

Thus the assertion is proved.

THEOREM 1. Suppose that a family of linear operators $\left\{A_{\varepsilon}\right\}(\varepsilon>0)$ and a closed linear operator $A$ from a Banach space $X$ to $X$ satisfy the following conditions:

(1) for some fixed number $\lambda$, the equation

$$
\left(\lambda-A_{\varepsilon}\right) y=x \quad(x \in X)
$$

has a unique solution $y=R\left(\lambda ; A_{\varepsilon}\right) x \in \mathscr{D}\left(A_{\varepsilon}\right)$ and $\left\|R\left(\lambda ; A_{\varepsilon}\right)\right\| \leqq K_{\lambda}$, where $K_{\lambda}$ is a positive number independent of $\varepsilon$,

(2) $\mathscr{D}\left(A_{\varepsilon}\right) \supset \mathscr{D}(A), \quad \overline{\mathscr{D}(A)}=X$,

(3) $A_{\varepsilon} x=A x+\varepsilon B_{\varepsilon} x \quad(x \in \mathscr{D}(A))$,

$$
\left\|B_{\varepsilon} x\right\| \leqq K(x) \quad(x \in \mathscr{D}(A)),
$$

where $K(x)$ is a positive number independent of $\varepsilon$.

Then we have $\mathscr{R}(\lambda-A)=(\lambda-A) \mathscr{D}(A)=X$.

Proof. It follows from Proposition 1 that the limit operator $R_{0}(\lambda ; A)$ exists and bounded on $\overline{\mathscr{R}}(\lambda-A)$.

Let $(\lambda-A) x_{n} \rightarrow y$ as $n \rightarrow \infty$. Then it follows from the boundedness of $R_{0}(\lambda ; A)$ that $x_{n} \rightarrow R_{0}(\lambda ; A) y$ and so that

$$
A x_{n} \rightarrow \lambda R_{0}(\lambda ; A) y-y
$$

as $n \rightarrow \infty$. Since $A$ is closed, $R_{0}(\lambda ; A) y \in \mathscr{D}(A)$ and $y \in \mathscr{R}(\lambda-A)$. Thus we have $\overline{\mathscr{R}}(\lambda-A)=\mathscr{R}(\lambda-A)$. It is easy to see that $\lambda-A_{\varepsilon}$ is closed and

$$
\begin{aligned}
\left(\lambda-A_{\varepsilon}\right) R_{0}(\lambda ; A) x & =(\lambda-A) R_{0}(\lambda ; A) x \\
& -\varepsilon B_{\varepsilon} R_{0}(\lambda ; A) x \quad(x \in \mathscr{R}(\lambda-A)) .
\end{aligned}
$$

Hence, from the closed graph theorem it follows that $B_{\varepsilon} R_{0}(\lambda ; A)$ is a bounded linear operator on $\mathscr{R}(\lambda-A)$. Moreover we have, for any $x \in \mathscr{D}(A)$,

$$
\left\|B_{\varepsilon} R_{0}(\lambda ; A)(\lambda-A) x\right\|=\left\|B_{\varepsilon} x\right\| \leqq K(x)<\infty .
$$

Using the resonance theorem it follows that there exists a positive number $L_{\lambda}$ which is independent of $\varepsilon$ such that 


$$
\left\|B_{\varepsilon} R_{0}(\lambda ; A)\right\|_{\mathscr{R}(\lambda-A)} \leqq L_{\lambda} .
$$

Consequently we obtain the basic relation, for any $x \in \mathscr{D}(A)$,

$$
\begin{aligned}
\left\|\varepsilon B_{\varepsilon} x\right\| & =\left\|\varepsilon B_{\varepsilon} R_{0}(\lambda ; A)(\lambda-A) x\right\| \\
& \leqq \varepsilon L_{\lambda}\|(\lambda-A) x\| \leqq \varepsilon L_{\lambda}\|A x\|+\varepsilon|\lambda| L_{\lambda}\|x\| .
\end{aligned}
$$

Thus the assertion follows from Remark 3.

REMARK 4. Let $A$ be a closed linear operator with dense domain $\mathscr{D}(A)$. Suppose that $A_{\varepsilon}=A+\varepsilon B$ generates a strongly continuous semi-group of linear contraction operators for every small $\varepsilon\left(0<\varepsilon<\varepsilon_{0}\right)$ and $\mathscr{D}\left(A_{\varepsilon}\right) \supset \mathscr{D}(A)$.

Then $A$ generates a strongly continuous semi-group of linear contraction operators.

Proof. Using Theorem 1 and Proposition 1, it follows from the Hille-Yosida theorem. (cf. [3], [11]).

2. The object of this section is to show that some special family of linear operators $\left\{A_{n}\right\}(n=1,2, \cdots)$ from a weakly complete Banach lattice $X$ to $X$ satisfies the convergence condition and to solve the problem on the perturbation theory for semi-groups of operators which is sited in the introductory part.

Let $X$ be a Banach lattice with a semi-order $\geqq$ and $[x, y](x, y \in X)$ denote a complex-valued (real-valued) function defined on $X \times X$ called a semi-inner product having the following properties (cf. [4], [6], [7]):

(1) $[x+y, z]=[x, z]+[y, z]$,

(2) $[\lambda x, y]=\lambda[x, y]$,

(3) $[x, x]=\|x\|^{2}$,

(4) $|[x, y]| \leqq\|x\|\|y\|$,

(5) if $y \geqq 0$, then $[x, y] \geqq 0$ for all $x \geqq 0$,

(6) $\left[x, x^{+}\right]=\left\|x^{+}\right\|^{2}$,

where $x^{+}=\sup (x, 0), x^{-}=\sup (-x, 0)$, and $|x|=\sup (x,-x)$.

The following theorem is essentially due to Reuter [8].

Proposition 5. Suppose that linear operators $A_{0}$ and $A_{1}$ on a Banach lattice $X$ satisfy the following conditions:

(1) for $n=0,1$ and some $\lambda>0$, the equation

$$
\left(\lambda-A_{n}\right) y=x \quad(x \in X)
$$

has a unique solution $y=R\left(\lambda ; A_{n}\right) x \in \mathscr{D}\left(A_{n}\right)$ and 


$$
R\left(\lambda ; A_{n}\right) x \geqq 0 \quad(x \geqq 0),
$$

(2) there exist dense subspaces $\mathscr{C}$ and $\mathscr{M}_{1}$ such that

$$
\begin{gathered}
A_{1} x \geqq A_{0} x \quad(x \geqq 0, x \in \mathscr{M}), \\
R\left(\lambda ; A_{1}\right) \mathscr{M}_{1} \subset \mathscr{C} .
\end{gathered}
$$

Then the following inequality holds:

$$
R\left(\lambda ; A_{1}\right) x \geqq R\left(\lambda ; A_{0}\right) x \quad\left(x \geqq 0, x \in \mathscr{C}_{1}\right)
$$

Proof. If $x \geqq 0$ and $x \in \mathscr{C}_{1}$, then $R\left(\lambda ; A_{1}\right) x \geqq 0$ and $R\left(\lambda ; A_{1}\right) x \in$ and thus we have

$$
\begin{gathered}
A_{1} R\left(\lambda ; A_{1}\right) x \geqq A_{0} R\left(\lambda ; A_{1}\right) x, \\
\left(\lambda-A_{0}\right) R\left(\lambda ; A_{1}\right) x \geqq\left(\lambda-A_{1}\right) R\left(\lambda ; A_{1}\right) x=x .
\end{gathered}
$$

Operating $R\left(\lambda ; A_{0}\right)$, we obtain

$$
R\left(\lambda ; A_{1}\right) x \geqq R\left(\lambda ; A_{0}\right) x .
$$

Let $\Sigma=\left\{T_{t} ; t \geqq 0\right\}$ be a one-parameter semi-group of linear operators from a Banach lattice $X$ to $X$ satisfying the following conditions:

(1) $T_{0} x=x, T_{t+s} x=T_{t} T_{s} x \quad(x \in X, t, s \geqq 0)$,

(2) $\left\|T_{t} x\right\| \leqq\|x\| \quad(x \in X, t \geqq 0)$,

(3) $\lim T_{t} x=x \quad(x \in X)$,

(4) $\quad \stackrel{t \rightarrow 0+}{T_{t}} x \geqq 0 \quad(x \geqq 0, t \geqq 0)$.

Such a semi-group is called a strongly continuous semi-group of positive contraction operators.

The following theorem is due to Phillips and is a variant of the Hille-Yosida theorem which will be convenient for purpose. (cf. [7]).

THEOREM. (Phillips). A necessary and sufficient condition for a linear operator $A$ with dense domain to generate a strongly continuous semi-group of positive contraction operators is that $\mathscr{R}(I-A)=X$ and that $A$ is dispersive, that is,

$$
\left\lfloor A x, x^{\dagger}\right\rfloor \leqq 0 \quad(x \in \mathscr{D}(A)) .
$$

THEOREM 2. Suppose that a family of linear operators $\left\{A_{n}\right\}$ $(n=1,2, \cdots)$ which generate strongly continuous semi-groups $\left\{\sum_{n}\right\}$ of positive contraction operators on a weakly complete Banach lattice $X$ satisfies the following conditions: there exist dense subspaces $\mathbb{C l}$, $\mathscr{C}_{0}$ and $\left\{\mathscr{C l}_{n}\right\}$ such that 
(1) $\lim _{n, n^{\prime} \rightarrow \infty}\left\|A_{n} x-A_{n^{\prime}} x\right\|=0 \quad(x \in \mathscr{C})$,

(2) $A_{n+1} x \geqq A_{n} x \quad\left(x \geqq 0, x \in \mathscr{C}_{n}\right)$,

(3) $R\left(\lambda ; A_{n}\right) \mathscr{M}_{0} \subset \mathscr{C}_{n}$,

(4) $\mathscr{A}_{0}^{+}=\left\{x^{+} ; x \in \mathscr{L}_{0}\right\} \subset \mathscr{L}_{0}$.

Then the limit operator $A=\lim A_{n}$ on $\mathscr{C l}$ has a closed extension $\widetilde{A}$ which generates a strongly continuous semi-group $\sum$ of positive contraction operators.

Moreover we have

$$
T_{t} x=\lim _{n \rightarrow \infty} T_{t}^{(n)} x \quad(x \in X, t \geqq 0),
$$

where $\sum_{n}=\left\{T_{t}^{(n)} ; t \geqq 0\right\}$ and $\sum=\left\{T_{t} ; t \geqq 0\right\}$.

Proof. By the Hille-Yosida theorem (cf. [3], [11]) we find that the conditions (1) and (2) in Proposition 5 and the following norm condition are satisfied for any pair $\left\{A_{n}, A_{n+1}\right\}$.

$$
\left\|R\left(\lambda ; A_{n}\right)\right\| \leqq \lambda^{-1}
$$

Thus we have, for any $n$,

$$
R\left(\lambda ; A_{n+1}\right) x \geqq R\left(\lambda ; A_{n}\right) x \quad\left(x \geqq 0, x \in \mathbb{C}_{0}\right) .
$$

Since $X$ is weakly complete, the norm condition and this inequality imply that there exists $y \geqq 0$ such that

$$
\lim _{n \rightarrow \infty}\left\|R\left(\lambda ; A_{n}\right) x-y\right\|=0 \text {. }
$$

From a representation of $x: x=x^{+}-x^{-}$, we have, for any $x \in \mathbb{L}_{0}$, using the condition (4),

$$
\lim _{n, n^{\prime} \rightarrow \infty}\left\|R\left(\lambda ; A_{n}\right) x-R\left(\lambda ; A_{n^{\prime}}\right) x\right\|=0
$$

and we have this convergence relation for all $x \in X$ by the condition $\overline{\mathscr{C}}_{0}=X$. We denote $\widetilde{R}(\lambda ; A)=\lim R\left(\lambda ; A_{n}\right)$. Then $\widetilde{R}(\lambda ; A)$ is positive and satisfies the norm condition $(*)$. The assertion is now proved by Theorem 2 in [1]. We sketch the proof of this theorem.

Since $R\left(\lambda ; A_{n}\right)$ satisfies the resolvent equation

$$
R\left(\lambda ; A_{n}\right)-R\left(\lambda^{\prime} ; A_{n}\right)=-\left(\lambda-\lambda^{\prime}\right) R\left(\lambda ; A_{n}\right) R\left(\lambda^{\prime} ; A_{n}\right)
$$

$\widetilde{R}(\lambda ; A)$ also does. Then we find that $\widetilde{R}(\lambda ; A)$ is a one-to-one transformation from $X$ to $\mathscr{R}(\widetilde{R}(\lambda ; A))$ and $\widetilde{A}_{\lambda}=\lambda-\widetilde{R}(\lambda ; A)^{-1}$ is independent of $\lambda$, that is,

$$
\widetilde{A} x=\widetilde{A}_{\lambda} x=\widetilde{A}_{\lambda^{\prime}} x \quad(x \in \mathscr{R}),
$$


where $\mathscr{R}=\mathscr{R}(\widetilde{R}(\lambda ; A))=\mathscr{R}\left(\widetilde{R}\left(\lambda^{\prime} ; A\right)\right)$.

Then, by the Hille-Yosida theorem, we find that $\widetilde{A}$ generates a strongly continuous semi-group of contraction operators. The positivity and the convergence of semi-groups are verified by the condition (**). It is readily verified that $\widetilde{A}$ is a closed extension of $A$.

REMARK 5. Suppose that a family of linear operators $\left\{A_{n}\right\}$ $(n=1,2, \cdots)$ which generate strongly continuous semi-groups of positive contraction operators on a weakly complete Banach lattice $X$ satisfies the following conditions:

(1) $\lim _{n, n^{\prime} \rightarrow \infty}\left\|A_{n} x-A_{n^{\prime}} x\right\|=0 \quad(x \in \mathscr{C l})$, where $\mathscr{C}$ is a dense subspace in $X$,

(2) $A_{n+1} x \geqq A_{n} x \quad\left(x \geqq 0, x \in \mathscr{D}\left(A_{n}\right)\right)$,

(3) $\mathscr{D}\left(A_{n+1}\right) \supset \mathscr{D}\left(A_{n}\right)$.

Then the assertion in Theorem 2 is true.

REMARK 6 . In Theorem 2, the condition (1) can be replaced by the following condition:

$$
\left\|A_{n}^{2} x\right\| \leqq K(x) \quad\left(x \in \mathscr{C}_{2}\right),
$$

where $K(x)$ is a positive number independent of $n$ and $\mathscr{C}_{2}$ is dense in $X$.

Proof. We remark that the convergence of the family of resolvents in Theorem 2 does not depend on (1). Then we have, for any $x \in \mathbb{L}_{2}$,

$$
\begin{aligned}
\left\|A_{n} x-A_{n^{\prime}} x\right\| \leqq & \lambda\left\|R\left(\lambda ; A_{n}\right) A_{n} x-R\left(\lambda ; A_{n^{\prime}}\right) A_{n^{\prime}} x\right\| \\
& +\left\|A_{n} x-\lambda R\left(\lambda ; A_{n}\right) A_{n} x\right\| \\
& +\left\|A_{n^{\prime}} x-\lambda R\left(\lambda ; A_{n^{\prime}}\right) A_{n^{\prime}} x\right\| \\
\leqq & \lambda^{2}\left\|R\left(\lambda ; A_{n}\right) x-R\left(\lambda ; A_{n^{\prime}}\right) x\right\| \\
& +\left\|R\left(\lambda ; A_{n}\right) A_{n}^{2} x\right\|+\left\|R\left(\lambda ; A_{n^{\prime}}\right) A_{n^{\prime}}^{2} x\right\| \\
\leqq & \lambda^{2}\left\|R\left(\lambda ; A_{n}\right) x-R\left(\lambda ; A_{n^{\prime}}\right) x\right\|+2 \lambda^{-1} K(x) .
\end{aligned}
$$

Letting $\lambda \rightarrow \infty$, we have, for any $\varepsilon>0$,

$$
\left\|A_{n} x-A_{n^{\prime}} x\right\| \leqq \lambda^{2}\left\|R\left(\lambda ; A_{n}\right) x-R\left(\lambda ; A_{n^{\prime}}\right) x\right\|+\varepsilon
$$

and the assertion is proved by (**).

From Remark 4 in [1] it follows that

REMark 7. Suppose that there exists a dense subspace $\mathscr{A}_{2}$ such that $\widetilde{R}(\lambda ; A) \mathscr{C}_{2} \subset \mathscr{M}$ in Theorem 2 , then $\widetilde{A}$ is the closure of $A$. 
We next concern with the generation of contraction semi-groups which dominate a given semi-group and give an alternative form of a theorem of Reuter, Miyadera and Olubummo (cf. [8], [5], [6], [7]).

Given a one-parameter semi-group $\sum=\left\{T_{t} ; t \geqq 0\right\}$ of positive contraction operators, if $\Sigma^{\prime}=\left\{T_{t}^{\prime} ; t \geqq 0\right\}$ is another one, we say that $\Sigma^{\prime}$ dominates $\Sigma$, if

$$
T_{t}^{\prime} x \geqq T_{t} x \quad(x \geqq 0, t \geqq 0) .
$$

In applications, it is important to know whether a given semigroup $\Sigma$ is dominated by any other semi-group $\Sigma^{\prime}$.

The following lemmas in a Banach space will be required.

Lemma. (Lumer and Phillips). If $A$ with dense domain is a dissipative operator, that is,

$$
\operatorname{Re}[A x, x] \leqq 0 \quad(x \in \mathscr{D}(A)),
$$

then $A$ has a closed extension.

Proposition 6. Suppose that a linear operator $A$ which generates a strongly continuous semi-group of contraction operators on a Banach space $X$ and a linear operator $B$ with domain $\mathscr{D}(B) \supset \mathscr{D}(A)$ satisfy the following condition: $A+B$ has a closed extension. Then

$$
\|B R(\lambda ; A)\| \leqq K
$$

where $K$ is a positive number independent of $\lambda>1$ and

$$
\lim _{\lambda \rightarrow \infty}\|B R(\lambda ; A) x\|=0 \quad(x \in X) .
$$

The proof of Proposition 6 is readily verified by using the resolvent equation and is omitted.

Theorem 3. In a weakly complete Banach lattice $X$ let $A$ be the generator of a positive contraction semi-group $\sum$ and let $B$ be a lenear operator with domain $\mathscr{D}(B) \supset \mathscr{D}(A)$. Then $A_{1}=A+B$ or its closed extension generates a positive contraction semi-group $\sum^{\prime}$ which dominates $\Sigma$ if and only if

$$
\begin{aligned}
& \operatorname{Re}\left[A_{1} x, x\right] \leqq 0 \quad(x \in \mathscr{D}(A)), \\
& B x \geqq 0 \quad(x \geqq 0, x \in \mathscr{D}(A)) .
\end{aligned}
$$

Proof. To prove the sufficiency of the conditions (1) and (2), we approximate $A_{1}$ by a sequence of linear operators $\left\{A_{n, \lambda}\right\}$ in the following way. Define a sequence of linear operators $\left\{A_{n, \lambda}\right\}$ by 


$$
A_{n, \lambda}=A+(n-\lambda) B R(n ; A) \quad(n \geqq \lambda)
$$

and $\left\{B_{n, \lambda}\right\}$ by

$$
\begin{aligned}
B_{n, \lambda} & =A_{n+1, \lambda}-A_{n, \lambda} \\
& =B R(n+1 ; A)(\lambda-A) R(n ; A) \quad(n \geqq \lambda) .
\end{aligned}
$$

Then it follows from Lemma (Lumer and Phillips) and Proposition 6 that there is a positive integer $L$ independent of $n$ and $\lambda$ such that $\left\|B_{n, \lambda}\right\| \leqq L$.

If we assume that the resolvent $R\left(\lambda ; A_{n, \lambda}\right)$ exists which acts on $X$ and is positive for some $\lambda$ and $n(n \geqq \lambda)$, then we have, for any $x \geqq 0$,

$$
\begin{aligned}
\lambda\left\|R\left(\lambda ; A_{n, \lambda}\right) x\right\|^{2}= & {\left[\lambda R\left(\lambda ; A_{n, \lambda}\right) x, R\left(\lambda ; A_{n, \lambda}\right) x\right] } \\
& \leqq\left[\lambda R\left(\lambda ; A_{n, \lambda}\right) x, R\left(\lambda ; A_{n, \lambda}\right) x\right] \\
& -\operatorname{Re}\left[A_{1} R\left(\lambda ; A_{n, \lambda}\right) x, R\left(\lambda ; A_{n, \lambda}\right) x\right] .
\end{aligned}
$$

Using Theorem (Phillips), we remark that $A$ is a dispersive operator. Thus we have

$$
\begin{aligned}
& \operatorname{Re}\left[A_{1} R\left(\lambda ; A_{n, \lambda}\right) x, R\left(\lambda ; A_{n, \lambda}\right) x\right] \\
&= \operatorname{Re}\left[A R\left(\lambda ; A_{n, \lambda}\right) x, R\left(\lambda ; A_{n, \lambda}\right) x\right] \\
&+\operatorname{Re}\left[B R\left(\lambda ; A_{n, \lambda}\right) x, R\left(\lambda ; A_{n, \lambda}\right) x\right] \\
&= {\left[A_{1} R\left(\lambda ; A_{n, \lambda}\right) x, R\left(\lambda ; A_{n, \lambda}\right) x\right] . }
\end{aligned}
$$

Hence we obtain

$$
\begin{aligned}
\lambda \| R(\lambda ; & \left.A_{n, \lambda}\right) x \|^{2} \\
& \leqq\left[\lambda R\left[\lambda ; A_{n, \lambda}\right) x, R\left(\lambda ; A_{n, \lambda}\right) x\right]-\left[A_{1} R\left(\lambda ; A_{n, \lambda}\right) x, R\left(\lambda ; A_{n, \lambda}\right) x\right] \\
& =\left[x, R\left(\lambda ; A_{n, \lambda}\right) x\right]-\left[B R(n ; A)(\lambda-A) R\left(\lambda ; A_{n, \lambda}\right) x, R\left(\lambda ; A_{n, \lambda}\right) x\right] \\
& \leqq\left[x, R\left(\lambda ; A_{n, \lambda}\right) x\right],
\end{aligned}
$$

where the last inequality holds by virtue of the formula

$$
\begin{aligned}
& (\lambda-A) R\left(\lambda ; A_{n, \lambda}\right) x \\
& \quad=x+(n-\lambda) B R(n ; A) R\left(\lambda ; A_{n, \lambda}\right) x .
\end{aligned}
$$

Thus we obtain, for any $x \geqq 0$ and then for any $x \in X$,

$$
\lambda\left\|R\left(\lambda ; A_{n, \lambda}\right) x\right\| \leqq\|x\| \text {. }
$$

By induction on $n$ we next show that the resolvent $R\left(\lambda ; A_{n, \lambda}\right)$ exists which acts on $X$ and is positive for any $\lambda>L$ and any $n \geqq \lambda$. It is clear that $R\left(\lambda ; A_{\lambda, \lambda}\right)=R(\lambda ; A)$ is a positive operator for any $\lambda>L$. Suppose that $R\left(\lambda ; A_{n, \lambda}\right)$ is positive for any $\lambda>L$ and some $n$, then we have $\left\|B_{n, \lambda} R\left(\lambda ; A_{n, \lambda}\right)\right\|<1$. It follows from this norm condition 
that $R\left(\lambda ; A_{n+1, \lambda}\right)$ exists which acts on $X$ and is given by the following formula (cf. [3], [11]):

$$
R\left(\lambda ; A_{n+1}, \lambda\right)=\sum_{k=0}^{\infty} R\left(\lambda ; A_{n, \lambda}\right)\left[B_{n, \lambda} R\left(\lambda ; A_{n, \lambda}\right)\right]^{k} .
$$

Since $B_{n, \lambda} R\left(\lambda ; A_{n, \lambda}\right)$ is positive, it follows that

$$
R\left(\lambda ; A_{n+1, \lambda}\right) x \geqq R\left(\lambda ; A_{n, \lambda}\right) x \geqq 0 \quad(x \geqq 0) .
$$

Hence, using the weakly completeness of $X$, we have for any $x \geqq 0$ and then $x \in X$,

$$
\lim _{n, n^{\prime} \rightarrow \infty}\left\|R\left(\lambda ; A_{n, \lambda}\right) x-R\left(\lambda ; A_{n^{\prime}, \lambda}\right) x\right\|=0 .
$$

To show that $\left\{R\left(\lambda^{\prime} ; A_{n, \lambda}\right) x\right\}\left(0<\lambda^{\prime}<\lambda\right)$ is also a Cauchy sequence for any $x \in X$, we make use of the relation

$$
R\left(\lambda-\mu ; A_{n, \lambda}\right)=\sum_{k=1}^{\infty} \mu^{k-1} R\left(\lambda ; A_{n, \lambda}\right)^{k},
$$

where, provided that $|\mu|<\lambda$, the right hand side converges uniformly in $n$ (cf. [3], [11]). It also follows from this formula that $\lambda^{\prime} R\left(\lambda^{\prime} ; A_{n, \lambda}\right)$ is positive and is a contraction operator for any $\lambda^{\prime}\left(0<\lambda^{\prime}<\lambda\right)$.

Let $k$ be a positive integer such that $k>L$. We define, for any $\lambda \leqq k$,

$$
\widetilde{R}\left(\lambda ; A_{k}\right) x=\lim _{n \rightarrow \infty} R\left(\lambda ; A_{n, k}\right) x \quad(x \in X) .
$$

Then it is easy to see that $\left\{\widetilde{R}\left(\lambda ; A_{k}\right) ; \lambda \leqq k\right\}$ satisfies the resolvent equation and the norm condition $\lambda\left\|\widetilde{R}\left(\lambda ; A_{k}\right)\right\| \leqq 1$.

Moreover $\left\{\widetilde{R}\left(\lambda ; A_{k}\right)\right\}_{k}$ is a consistent family of resolvents in the following sense:

$$
\widetilde{R}\left(\lambda ; A_{k^{\prime}}\right) x=\widetilde{R}\left(\lambda ; A_{k}\right) x \quad\left(x \in X, \lambda<k<k^{\prime}\right) .
$$

In fact, we have the inequality

$$
\begin{aligned}
\| \widetilde{R}(\lambda ; & \left.A_{k^{\prime}}\right) x-\widetilde{R}\left(\lambda ; A_{k}\right) x \| \\
\leqq & \left\|\widetilde{R}\left(\lambda ; A_{k^{\prime}}\right) x-R\left(\lambda ; A_{n, k^{\prime}}\right) x\right\| \\
& +\left[1+\lambda^{-1}\left(k^{\prime}-k\right) L\right]\left\|R\left(\lambda ; A_{n, k}\right) x-\widetilde{R}\left(\lambda ; A_{k}\right) x\right\| \\
& +\lambda^{-1}\left(k^{\prime}-k\right)\left\|B R(n ; A) \widetilde{R}\left(\lambda ; A_{k}\right) x\right\|
\end{aligned}
$$

and letting $n \rightarrow \infty$, we obtain the desired result.

Since $\left\{\widetilde{R}\left(\lambda ; A_{k}\right)\right\}_{k}$ is consistent, we have a family of resolvents

$$
\left\{\widetilde{R}\left(\lambda ; A_{1}\right)\right\} ; \widetilde{R}\left(\lambda ; A_{1}\right)=\widetilde{R}\left(\lambda ; A_{k}\right) \quad(\lambda \leqq k)
$$

which satisfies the norm condition $\lambda\left\|\widetilde{R}\left(\lambda ; A_{1}\right)\right\| \leqq 1$. 
Then, using the same method as that in the proof of Theorem 2, we find that $\widetilde{A}_{1}=\lambda-\widetilde{R}\left(\lambda ; A_{1}\right)^{-1}$ generates a strongly continuous semigroup $\Sigma^{\prime}$ of positive contraction operators which dominates $\Sigma$ and that $\widetilde{A}_{1}$ is a closed extension of $A_{1}$.

We now prove the inverse part. Let $\Sigma=\left\{T_{t} ; t \geqq 0\right\}$ and $\Sigma^{\prime}=$ $\left\{T_{t}^{\prime} ; t \geqq 0\right\}$. Then the condition (1) follows from

$$
\begin{aligned}
\operatorname{Re}\left[A_{1} x, x\right] & =\lim _{t \rightarrow 0+} \operatorname{Re}\left[t^{-1}\left(T_{t}^{\prime} x-x\right), x\right] \\
& =\lim _{t \rightarrow 0+} t^{-1} \operatorname{Re}\left\{\left[T_{t}^{\prime} x, x\right]-[x, x]\right\} \\
& \leqq 0 \quad(x \in \mathscr{D}(A)),
\end{aligned}
$$

and (2) follows from

$A_{1} x=\lim _{t \rightarrow{ }^{+}} t^{-1}\left(T_{t}^{\prime} x-x\right) \geqq \lim _{t \rightarrow 0+} t^{-1}\left(T_{t} x-x\right)=A x \quad(x \geqq 0, x \in \mathscr{D}(A))$.

Thus the assertion is proved.

REMARK 8. In Theorem 3 any one of the following conditions can take the place of the condition (1).

$$
\left[A_{1} x, x\right] \leqq 0 \quad(x \geqq 0, x \in \mathscr{D}(A))
$$

and $A_{1}$ has a closed extension,

$$
\left[A_{1} x, x\right] \leqq 0 \quad(x \geqq 0, x \in \mathscr{D}(A))
$$

and $B R(\lambda ; A)$ is a bounded linear operator for any $\lambda>0$.

The contents of this section will be discussed in [2] by virtue of the notation of Gâteaux differentials.

The author wishes to express his gratitude to Professor Isao Miyadera for his valuable advice.

\section{REFERENCES}

1. M. Hasegawa, A note on the convergence of semi-groups of operators, Proc. Japan Acad. 40 (1964), 262-266.

2. - On contraction semi-groups and (di)-operators (in preparation)

3. E. Hille and R. S. Phillips, Functional Analysis and Semi-groups, American Mathematical Society, 1957.

4. G. Lumer and R. S. Phillips, Dissipative operators in a Banach space, Pacific J. Math. 11 (1961), 679-698.

5. I. Miyadera, $A$ note on contraction semi-groups of operators, Tôhoku Math. J. 11 (1959), 98-105.

6. A. Olubummo, A note on perturbation theory for semi-groups of operators, Proc. Amer. Math. Soc. 16 (1964), 818-822.

7. R. S. Phillips, Semi-groups of positive contraction operators, Czechoslovak Math. J. 12 (1962), 294-313. 
8. G. E. H. Reuter, $A$ note on contraction semi-groups, Math. Scand. 3 (1955), 275-280.

9. H. F. Trotter, Approximation of semi-groups of operators, Pacific J. Math. 8 (1958), 887-919.

10. - On the product of semi-groups of operators, Proc. Amer. Math. Soc. 10 (1959), 545-551.

11. K. Yoshida, Functional Analysis, Springer, Berlin, 1965.

Received October 27, 1965.

WASEDA UNIVERSITY, TOKYO 



\section{PACIFIC JOURNAL OF MATHEMATICS}

\section{EDITORS}

H. SAMELSON

Stanford University

Stanford, California

J. P. JANS

University of Washington

Seattle, Washington 98105
J. DugundJI

University of Southern California

Los Angeles, California 90007

RICHARD ARENS

University of California

Los Angeles, California 90024

\section{ASSOCIATE EDITORS}

E. F. BECKENBACH

B. H. NeumanN

F WolF

K. YOSIDA

\section{SUPPORTING INSTITUTIONS}

UNIVERSITY OF BRITISH COLUMBIA

CALIFORNIA INSTITUTE OF TECHNOLOGY

UNIVERSITY OF CALIFORNIA

MONTANA STATE UNIVERSITY

UNIVERSITY OF NEVADA

NEW MEXICO STATE UNIVERSITY

OREGON STATE UNIVERSITY

UNIVERSITY OF OREGON

OSAKA UNIVERSITY

UNIVERSITY OF SOUTHERN CALIFORNIA
STANFORD UNIVERSITY

UNIVERSITY OF TOKYO

UNIVERSITY OF UTAH

WASHINGTON STATE UNIVERSITY

UNIVERSITY OF WASHINGTON

AMERICAN MATHEMATICAL SOCIETY CHEVRON RESEARCH CORPORATION TRW SYSTEMS

NAVAL ORDNANCE TEST STATION

Printed in Japan by International Academic Printing Co., Ltd., Tokyo Japan 


\section{Pacific Journal of Mathematics}

\section{Vol. 21, No. $1 \quad$ November, 1967}

Friedrich-Wilhelm Bauer, Der Hurewicz-Satz................... 1

D. W. Dubois, A note on David Harrison's theory of preprimes . ......... 15

Bert E. Fristedt, Sample function behavior of increasing processes with stationary, independent increments ..................... 21

Minoru Hasegawa, On the convergence of resolvents of operators....... 35

Søren Glud Johansen, The descriptive approach to the derivative of a set function with respect to a $\sigma$-lattice ....................... 49

John Frank Charles Kingman, Completely random measures ............ 59

Tilla Weinstein, Surfaces harmonically immersed in $E^{3} \ldots \ldots \ldots \ldots \ldots . . \ldots 9$

Hikosaburo Komatsu, Fractional powers of operators. II. Interpolation spaces ......................................... 89

Edward Milton Landesman, Hilbert-space methods in elliptic partial differential equations ...................................... 113

O. Carruth McGehee, Certain isomorphisms between quotients of a group algebra ........................................ 133

DeWayne Stanley Nymann, Dedekind groups .................. 153

Sidney Charles Port, Hitting times for transient stable processes ......... 161

Ralph Tyrrell Rockafellar, Duality and stability in extremum problems involving convex functions . ............................ 167

Philip C. Tonne, Power-series and Hausdorff matrices . . .............. 189 